\section{EFFICACY OF PLATELET RICH PLASIMA AS MONOTHERAPY IN TREATMENT OF ALOPECIA AREATA.}

KEY WORDS: Alopecia

Areata , platelet rich plasma Growth factors, SALT score

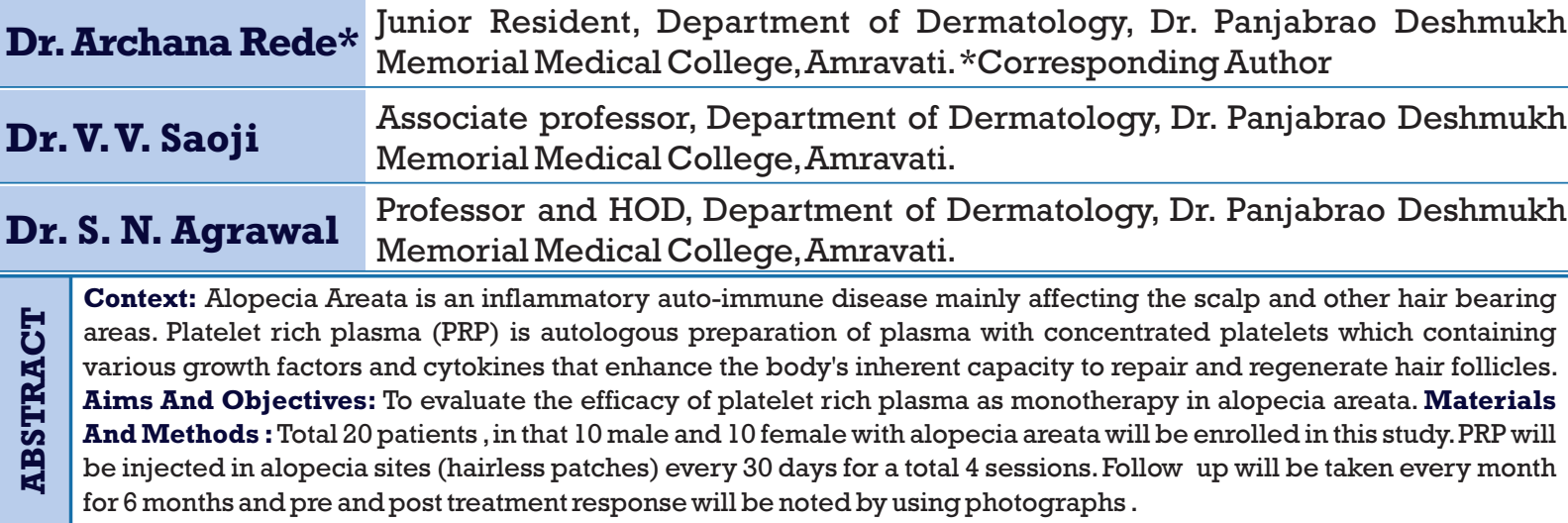

\section{Introduction-}

Alopecia Areata ( $\mathrm{AA})$ is an inflammatory auto-immune disease mainly affecting the scalp and other hair bearing areas. Alopecia areata was first recognized as a skin disese by the Ebers Papyrus in 1500-2500 BC .Meanwhile, the term alopecia was first used by sauvages in 1760 , which was then published by Lyons in $1760 .^{(1,2)}$ Based on an epidemiological point of view, this disease affects $0.2 \%$ of the total world population. This condition can manifest in the form of small circular patches of hair that fall out to diffuse patches. The area often affected is the scalp. ${ }^{(2,3)}$

The exact etiology and pathogenesis of $\mathrm{AA}$ are currently not fully understood. However, it is believed that the condition is an autoimmune process through the autoreactive of CD8 $+\mathrm{T}$ cells. ${ }^{(4)}$

Alopecia areata affects the patients physically, psychologically And cosmetically ${ }^{(2)}$ Treatment of $A A$ is not curative ,but controls or limits the pathogenesis process .Most treatments are immunosuppressive or immunomodulatory. Intralesional corticosteroid injection is the most commonly used treatment .Based on overall treatments , the results have not been satisfactory, and the response to treatment varies between individuals. ${ }^{(5-7)}$

Recently, PRP has been used in aesthetics and dermatology fields. ${ }^{(6,7)}$ especially for wound repair. ${ }^{(8)}$ Use of PRP for AA was reported without an allergic reaction. Platelet rich plasma (PRP) is autologous preparation of plasma with concentrated platelets which containing various growth factors and cytokines that enhance the body's inherent capacity to repair and regenerate hair follicles.

\section{AIMS AND OBJECTIVES :}

To evaluate the efficacy of platelet rich plasma as monotherapy in alopecia areata .

\section{MATERIALS AND METHODS :}

This is a prospective study that was conducted at Dr. Panjabrao Deshmukh Medical College and Hospital, Amravati from January 2021 to June 2021 in which total 20 patients , in that 10 male and 10 female with alopecia areata were enrolled in this study.

\section{Treatment protocol :}

Method to prepare PRP: - Collect $10-20 \mathrm{ml}$ of patient's blood and mix it with anticoagulant such as Acid Citrate Dextrose (ACD). Platelet Rich Plasma prepared by two stage of centrifugation process.

2 stage centrifugation process :

\section{First Centrifugation [Soft Spin]:}

Approximately $1500 \mathrm{rpm}$ for $5 \mathrm{~min}$, which separate blood into 3 layers, namely, bottom most RBCs layer, topmost acellular plasma layer [platelet poor plasma] and intermediate PRP. Using sterile syringe, platelet poor plasma , PRP and some RBCs transferred into another tubes without anticoagulant. And these tubes was undergo second centrifugation.

\section{Second Centrifugation [Hard Spin]:}

second centrifugation started at $2500 \mathrm{rpm}$ for $15 \mathrm{~min}$. This allowed the platelets to settle at bottom of tubes with very few RBCs. Platelet poor plasma was form at the top of the tube. Most of the platelet poor plasma removed and discarded. Remaining PRP collected into insulin syringe.

PRP injected in alopecia sites (hairless patches) every 30 days for a total 4 sessions. Follow up was taken every month for 6 months and pre and post treatment response noted by using photographs.

\section{Inclusion Criteria:}

1) patients in the age group 10-50 years .

2) patients with disease stability of atleast one year duration

3) Alopecia areata Patient's without nail changes .

\section{Exclusion Criteria :}

1)Patients not willing to participate in the study

2) Patients with certain blood and bleeding disorders

3) Patients with keloid developing tendancy .

\section{RESULTS -}

Of 20 patients , only 2 patients had relapse. None of the patients had any side effects and all of them tolerated the procedure well.

\section{CONCLUSION :}

Alopecia areata is form of autoimmune hair loss without scarring, which affect the scalp with or without affecting the body . PRP therapy was introduced as an adjuvant therapy modality in AA .This therapy is easy to do and effective in Alopecia areata, without side effects .

\section{Strong Factor-}

PRP treatment for Alopecia areata is effective, safe and easy to perform procedure. 


\section{Limitation -}

Procedure is relatively costly, painful and time consuming .hence we did this study in small population.

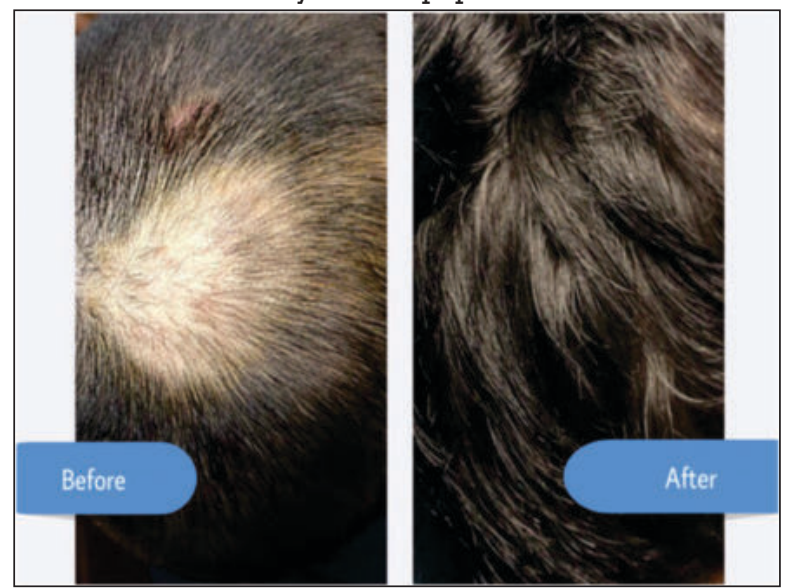

Fig. 1: Clinical picture of Alopecia Areata over parietal region of scalp. Before treatment and After $4^{\text {th }}$ session of PRP

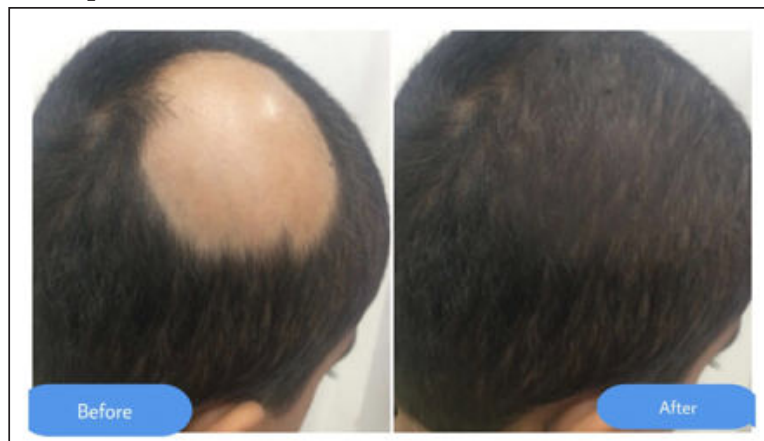

Fig. 2 : Clinical picture of Alopecia Areata (in 13 Year Old Child) over parietal region of scalp. Before treatment and After $4^{\text {th }}$ session of PRP

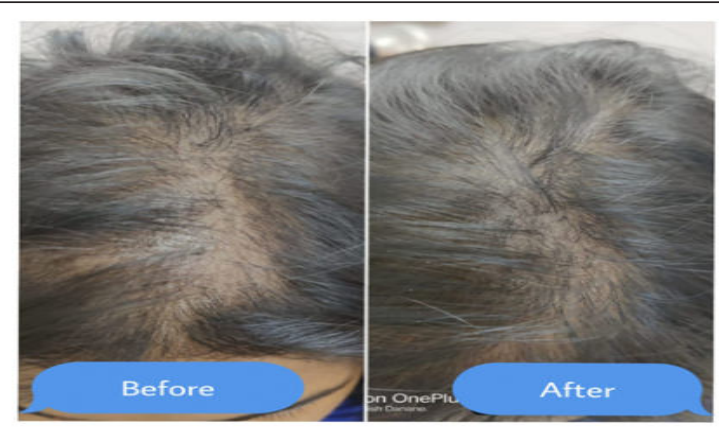

Fig. 3 : Clinical picture of Alopecia Areata (in 30 Year Old Female) over parietal region of scalp. Before treatment and After $4^{\text {th }}$ session of PRP

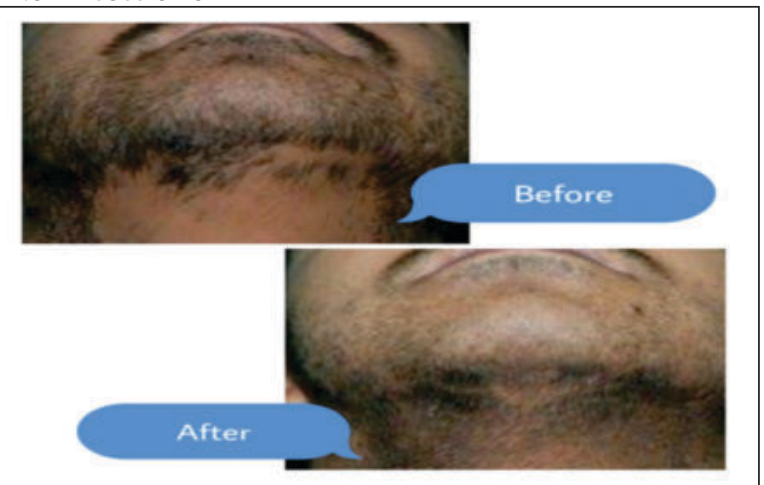

Fig. 4 : Clinical picture of Alopecia Areata over beard area. Before treatment and After $4^{\text {th }}$ session of PRP

|www.worldwidejournals.com |

\section{REFERENCES}

1. Otberg N, Shapiro J. Non-Scarring Focal Hair Loss. In: Hair Growth Disorders. Goldsmith LA, Katz SI, Gilchrest BA, Paller AS, Leffell DJ, Wolff K, editors. Fitzpatrickâ $€^{\mathrm{TM}} \mathbf{s}$ dermatology in general medicine, 8th ed. New York: McGraw-Hill;2012.991-994.

2. Amin SS, Sachdewa S. Alopecia areata:A review. Journal of the Saudi Society of Dermatology and Dermatologic Surgery. 2013;17:37-45.

3. Hordisky MK. Overview of Alopecia Areata. The society of Investigative Dermatology. 2013;16:1-2.

4. Signal A, Sonthania S, Verma P. Female Pattern Hair Loss. Indian Journal of Dermatology,Venereology and Leprology.2013;79(5):627-640.

5. Ovidio RP. Alopecia Areata: A News On Diagnosis, Pathogenesis, and Treatment. G Ital Dermatology Venereology. 2014;149:25-45.

6. Kumar A, Sharma RP. Role of Platelet Rich Plasma Therapy in Alopecia AreataA Prospective Study. International Journal of Contemporary Medical Research. 2016;3(8):2499-2502.

7. Langer C, Mahajan V. Platelet-Rich Plasma in Dermatology. JK Science. 2014;16(4):147-149.

8. Labusca LS, Cionca D. Clinical Review about the Role Of Platelet Rich Plasma for the Treatment of Traumatic and Degenerative Musculoskeletal Disorders. Orthopedics and Rheumatology Journal.2016;2(3):1-9.

9. Wang E, Kevin J. Etiopathogenesis of Alopecia Areata; Why Do Our Patients Get It.Dermatologic Therapy.2011;24:337-347.

10. Li ZJ, Choi HI, Choi DK, Sohn KC, Im M, SeoY, LeeYH, Lee JH, LeeY. Autologous platelet-rich plasma: a potential therapeutic tool for promoting hair growth. Dermatol Surg. 2012;38(7 Pt 1):1040-6.

11. Montero EC, Santos F, Fernadez S. Platelet-Rich Plasma: Application in Dermatology. Actas Dermosifiliogr.2015;106(2):104-111.

12. Marlovits S, Mousavi M. Gabler C, Erdos J, Vecsei V. A new simplified technique for producing platelet-rich plasma: a short technical note. Eur Spine J.2004;13(Suppl 1):S102-106.

13. Uebel CO, Escobar PD. Follicular Unit Megassesions and Platelet Growth Factor. Rev Bras Ur Plast. 2013;281:154-164.

14. Sammeling CE, Heyneman A. The Use of Platelet-Rich Plasma in Plastic Surgery, A Systemic Review. Journal of Plastic Reconstruction and Aesthetic Surgery. 2013;66:301-312.

15. Gentile P, Garcivich S, Bielli A. The Effect of Platelet Rich Plasma in Hair Regrowth ; A Randomized Placebo-Controlled Trial.Stem Cells Translational Medicine. 2015;4:1317-1323.

16. Lynch Md, Baschirs. Application of Platelet-Rich Plasma in Dermatology: a Critical Appraisal of The Literature. Journal of Dermatology Treatment. 2016;27(3):283-289

17. Fukaya M. Ito A. A New Economic Method for Preparing Platelet-rich Plasma. Plast Reconstr Surg Glob Open. 2014;2(6): 1-7.

18. Singhal P, Agarwal S, Dhot PS, Sayal SK. Efficacy of platelet-rich plasma in treatment of androgenic alopecia. Asian JTransfus Sci.2015;9(2):159-62.

19. Cervelli V, Garcovich S, Bielli A, Cervelli G, Curcio BC, Scioli MG, Orlandi A, Gentile P. The effect of autologous activated platelet-rich plasma (AA-PRP) injection on pattern hair loss: clinical and histomorphometric evaluation. Biomed Res Int. 2014:2014:760709.

20. Maria-Angeliki G, Alexandros-Efstratios K, Dimitris R, Konstantinos K. Platelet-rich Plasma as a Potential Treatment for Noncicatricial Alopecias. Int JTrichology. 2015 Apr-Jun; 7(2):54-63.

21. Messura MR, Nagata MJ. A Standardized Research Protocol for Platelet-Rich Plasma (PRP) Preparation in Rats. Original Research Article. 2011:8(3):299304.

22. Maazoca DA, Beth M. Platelet-Rich Plasma Differs According to Preparation Method and Human Variability. The Journal of Bone and Joint Surgery. 2012;94(4):308-316

23. Dhillion SR, Schaward EM. Platelet-Rich Plasma Therapy Future or Trend. Arthritis Research and Therapy. 2012;14:1-10.

24. Sommeling CE, Heyreman A. The Use of Platelet-Rich Plasma in Plastic Surgery:A Systematic Review.J Plast Reconstr Aesthet Surg. 2013;66:301-313.

25. Cole BJ, Soreyer ST. Platelet-Rich Plasma:Where Are Now And Where Are We Going. Sport Health Medicine. 2010;10(10):1-8.

26. Mubki T. Platelet-rich plasma combined with intralesional triamcinolone acetonide for the treatment of alopecia areata: A case report. Journal of Dermatology and Dermatology Surgery. 2016;20:87-90.

27. Uzzaif M, Vaaruni R. A Comparative Study of Treatment Modalities in Female Alopecia Areata. International Journal of Research in Medical Sciences. 2016;4(4):1229-1236. 